\title{
Subcarrier Allocation Techniques in OFDMA
}

\author{
G.Kavitha, N.Priya, R.Velvizhi
}

Abstract: Orthogonal Frequency Division several Access (OFDMA) is a access that is numerous where the regularity musical organization is divided in to range subcarriers and each individual is assigned a subset of this subcarriers to be used. Although the OFDMA framework provides a mechanism for a person to distribute information throughout the pair of assigned sub-carriers, it nevertheless departs the appropriate concern of just how to designate sub-carriers to users. The subcarrier resource allocation issue is complex for both charged energy minimization along with price maximization both in uplink and downlink situations. The allocation issue has gotten active desire for the $r$ esearch community and has now been examined from, essentially, two views: schemes that look for to attenuate the quantity of send energy and the ones that look for to obtain throughput that is optimum. Analysis a few of the crucial algorithms which were proposed for efficient subcarrier allocation is completed in their paper.

Keywords: OFDMA; subcarrier, price and energy allocation; fairness consideration

\section{INTRODUCTION}

Orthogonal Frequency Division several Access (OFDMA) can be viewed as an expansion of Orthogonal Frequency Division Multiplexing (OFDM) to situations which are multi-user. OFDM is an effective modulation that is electronic which divides the transmitted bitstream into numerous substreams and giving these bitstreams over $\mathrm{d}$ ifferent orthogonal subcarriers [1-5]. In OFDM, the information price achieved $\mathrm{o} n$ each subcarrier is dramatically not as much as the total $\mathrm{d}$ ata price, but, the bandwidth occupied by each subcarrier is significantly lower than the machine bandwidth that is total. The quantity of subcarriers is chosen so that each subcarrier has a bandwidth significantly less than the coherence bandwidth regarding the channel in o rder for the subcarriers to have fading that is fairly flat. This enables OFDM to effectively resist towards the impacts of regularity fading that is selective the price and energy are modified for each subcarrier separately. The subcarriers in OFDM aren't necessary to be contiguous like in Fig. 1. Therefore, a big constant block of range is not required for higher rate multicarrier communications, alternatively, a few contiguous obstructs of smaller size can be utilized hence attaining freedom in range allocation a spectrum administration that is nd.

A significant benefit of OFDM is loading that is adaptive in the adaptive modulation methods. The concept that is fundamental to alter the information price and energy assigned every single subcarrier according tothe channel gain of this subcarrier. Thus, the charged energy and price connected with each subcarrier could be optimized to

Revised Manuscript Received on July 22, 2019.

G.Kavitha, Department of CSE, Bharath Institute of Higher Education and Research

N.Priya, Department fCE, Bharath Institute of Higher Education and Research and Research
R.Velvizhi, Department of CSE, Bharath Institute of Higher Education

increase the price for confirmed optimum send energy or even to minmize the transfer power for a desired minimum price [6-8].

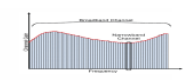

Figure 1: Channel variation on the subcarriers which can be various.

OFDMA can be considered as an expansion of OFDM tomulti-user s cenarios. S ection II outlines OFDMA technology. In Section III, subcarrier allocation schemes are explained. Various subcarrier allocation techniques are described in part IV and also the paper is determined with Section V.

\section{OFDMA}

OFDMA is certainly one when the regularity musical organization is split into range subcarriers and every individual is assigned a subset associated with subcarriers for use. Each subcarrier is assigned solely to at least one user i.e., the companies are not provided among users. These subset of subcarriers is named subchannel. The subcarriers u se certain frequencies to be able to be entirely orthogonal to one another [9-11]. OFDMA allocates these orthogonal subcarriers to $u$ that is significantly diffent. The synthesis of subchannels from subcarriers is an concept that is essential OFDMA systems. The development may be categorized into two kinds; one is mapping o $\mathrm{f}$ a contiguous band of subcarriers into a subchannel called once the Adjacent Sub carrier technique (ASM) and also the o ther could be the iversity that is d grouping called as Diversity Subcarrier Method (DSM) wherein the subchannel typically contains non contiguous subcarriers. Orthogonal regularity unit access that is multipleOFDMA) exploits multiuser d iversity, rendering it appealing for mobile broadband cordless access systems such as for example LTE and WiMAX. Consequently, radio resource administration, composed of efficient energy and subcarrier allocation, whether into the up website link or downlink, is important in using the many benefits of OFDMA [12-16].

In multiuser systems u sing fixed time-division access that is multipleTDMA) or frequency-division multiple access (FDMA) as multiaccess schemes, e ach user is allocated a predetermined time slot or regularity musical organization to utilize OFDM with adaptive modulation. In Fig. 2 , the full time regularity view of a t OFDMA that is ypical sign shown for an incident where you will find three users. It may be seen from Fig. 2 that user's signals are divided both within the time-domain making use of various OFDM s ymbols as well as in the subcarrier domain. Therefore, both right time and regularity resources are accustomed to support multiuser transmissions [17-19]. 


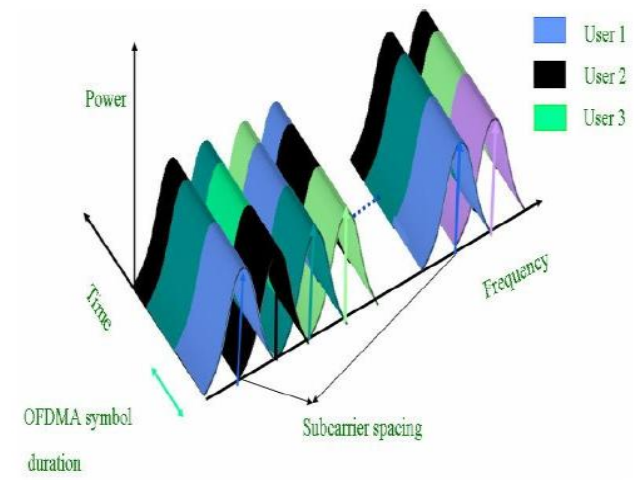

Figure 2: Time-Frequency view of a OFDMA that is typical signal] Here are generally subcarriers which encounter deep fade

And are also perhaps not energy efficient to hold any provided information bit [20-25]. These subcarriers are maybe not utilized to carry information.

Consequently, these subcarriers that are unused the allocatedTime regularity or slot musical organization of a person are squandered and are also maybe not employed by other users. Nevertheless, the subcarriers which can be found inDeep fade to 1 individual may possibly not be in deep fade for any other users. In reality, it's q uite not likely that a subcarrier will be in deep fade for many users, whilst the diminishing parameters for various users are mutually separate. This process shall enable all theSubcarriers to be utilized better because a subcarrier will be kept unused only when it's in deep fade to all or any users. Effectiveness of resource utilization can notably be enhanced by applying strategies that exploit the independency of fading processes for numerous users in the system [26]. Thus OFDMA exploits multiuser that is $\mathrm{d}$.

Assigning subcarriers to your users just isn't an age task that is asy. Current studies explored the situation of finding an subcarrier that is optimal energy allocation techniques. The resource allocation issue is, in every time slot, offered $\mathrm{m}$ users $\mathrm{n}$ subcarriers and channel that is complete, just how to designate disjoint sets of subcarriers every single individual in order to optimize system goal. Efficient subcarrier allocation can be carried out by minimizing the transfer that is total while keeping the info rates $[27,28]$.

\section{SUBCARRIER ALLOCATION SCHEMES}

The resource allocation issue is examined essentially fromtwo perspectives; schemes that look for to attenuate quantity of transfer power (Margin Adaptive) and schemes that look for to maximise throughput (Rate Adaptive) [29, 30]. There are two main goals for energy minimization; minmise overall energy usage and reduce the $u$ serpower usage that is maximal. Additionally there are two main objectives for price maximization; optimize overall transmission price and optimize $u$ ser transmission price that is minimal.

Taking into consideration the objective for the optimization issue in subcarrier allocation, algorithms are categorized into two teams: "margin adaptive" and adaptive" that is"rate. The aim that is primary margin adaptive algorithms is always to minimise the sum total send energy for the system while keeping each individual along with its necessary quality of solution demands; such as for example information price and bit mistake price (BER). The key goal in price adaptive algorithms is optimize the sum total system throughput because of the constraint in the send $\mathrm{p}$ ower that is total [31-35].

For basic uplink access that is numerous, price maximization algorithms are offered primary focus into the literary works, since energy minimization people are of less value considering the fact that minimizing the full total send energy of all userswhich can be running on various batteries bears no significant and thus is real. It was seen that allocating subcarriers toThe users with gain that is greatest after which allocating the sum total capacity to the subcarriers in accordance with waterfilling maximize throughput of the machine. [36-38]. Allocating subcarriers to your high-subcarrier-gain users impose unfairness in the users being low-subcarrier-gain. To eliminate this unfairness, fairness on the list of users must $\mathrm{b}$ age considered.

There are two main classes of price algorithms being adaptive throughput oriented and fairness oriented. The throughput-orientedsubcarrier allocation algorithms maximize the throughput that is instantaneous of system. Throughput-oriented algorithms lose the fairness within the functional system to optimize the throughput. In contrary to throughput-oriented algorithms, fairness-orientedalgorithms optimize the fairness that is instantaneous the users into the system. The target that is main throughput-oriented subcarrier allocation is optimize the total $\mathrm{d}$ ata price of multi-user OFDM system without considering the fairness. Throughput-orientedalgorithm is often named greedy algorithm. Fairness- oriented algorithm maximizes the fairness one of the users within the system by making the most of the information price associated with the individual that is minimum-rate each step of the process of subcarrier allocation. Within the action that is first on age subcarrier is allotted to each individual in which the channel gain may be the greatest forthat user. Next action, the consumer with all the information price that is cheapest is prioritized for subcarrier allocation. The process continues until most of the subcarriers are allocated. Since the algorithm attempts to optimize the information rates regarding the users aided by the information that is minimal in each step of the process, it's also called Max-Min subcarrier allocation algorithm. Throughput and fairness are ho $\mathrm{p}$ that is wever conflicting metrics. To increase the throughput, system will allocate more subcarriers towards the individual with greater subcarrier gain which might cause radio resource monopolization by lots that is little of sers, resulting in unfairness. Ther hand, to keep up the fairness, the machine will need to allocate more resources to your users in even worse channel conditions and; because of this, the machine throughput will degrade consequently in the o. As previously mentioned previous, the greatest objective in throughput-oriented algorithms would be to attain the po that is biggest ssible throughput. The primary goal would be to keep up with the fairness one of the users in fairness-oriented algorithms. The trade-off between $t$ fairness and hroughput must certanlybe considered. Therefore generally speaking, you can find three primary goals for radio resource allocation; making the most of throughput, minimizing transmission power and maintaining fairness among the list of users. The part that is after an evaluation of subcarrier allocation techniques for OFDMA.

\section{IV.VARIOUS SUBCARRIER}




\section{ALLOCATION METHODS IN OFDMA}

The approach that is simplest to subcarrier assignment would be toDisregard channel allocate and information companies to users proportional for their price demands. Subcarriers may be allocated in consecutive chunks (bands) or interleaved to boost regularity variety.

Among the widely used ways to designate subcarriers to users is a "water filling" technique which provides each subcarrier towards the individual with gain that is greatest onto it. This technique assigns price and energy optimally among subcarriers. Even though this technique provides energy that is optimal through a spectrally shaped channel for supplying its maximal capability, it doesn't help minimal price demands for specific $u$ sers. The essential point can be that the water pouring concept does not offer fairness $b$ etween the users with regards to the bit prices since it constantly assigns a subcarrier towards the individual because of the channel conditions which are most readily useful. Further, the computationalost that is $\mathrm{c}$ of algorithm is high [39].

Another way for eliminating the nagging dilemma of subcarrier allocation is CDMA over numerous companies (MC-CDMA). But we could observe that OFDMA o utperforms MC-CDMA because in OFDMA, in the place of transmitting over all subcarriers, users can select a channel that is most beneficial through the available stations.

- Multiuser OFDM with Adaptive Subcarrier, Bit, and energy Allocation:

Presuming knowledge of the channel that is instantaneousgains for many users, Wong et al. [5] propose a multiuser OFDM subcarrier, bit, and energy allocation algorithm to reduce the transfer energy that is total. This is carried out by assigning each individual a couple of subcarriers and also by determining the real range bits therefore the send energy degree for every single subcarrier.

There are typically subcarriers which encounter deep fade and therefore are perhaps not energy efficient to hold any offered information bit. Consequently, these subcarriers inside the allocated time regularity or slot musical organization of a person and they are squandered and generally are maybe not utilized by other users. Nonetheless, the subcarriers which come in deep fade to 1 individual may not $b$ age in d eep fade for any other users. In reality, it's quite not likely that a subcarrier will be in deep fade for many users, since the diminishing parameters for various users are mutually separate. This inspired the writers to take into account an multiuser that is adaptive allocation scheme in which the subcarriers are assigned to your users predicated on instantaneous channel information. This process allows all of the subcarriers to effortlessly be utilized more because a subcarrier may be kept unused only when it's in deep fade to any or all users. The send energy amounts should be changed appropriately as various subcarriers encounter different fades and transfer different nu mbers of bits.

They've considered a multiuser s ubcarrier, bit, and energy allocation scheme where all users transfer in most the best time slots. Their goal ended up being to reduce the transfer that is general by allocating the subcarriers to your users and also by determining the amount of bits as well as the power degree sent for each subcarrier based in the instantaneous diminishing traits of all of the users. The writers proposed an iterative algorithm to perform the multiuser subcarrier allocation inside their paper. After the subcarrier allocation is determined based on known instantaneous channel information, then solitary individual bit and energy allocation algorithm is placed on each individual on its allocated subcarriers to attenuate general total transmit energy that's needed is.

Based on their outcomes, some great benefits of this technique are; the algorithm outperforms other multiuser systems which can be OFDM OFDM-TDMA and OFDM-FDMA which use fixed and predetermined timeslot or subcarrierallocation schemes. Additionally there is certainly enhancement when it comes to general send that's needed is, bit mistake price (BER)Or the particular section of protection for a provided outage likelihood.

Because the algorithm makes use of leisure that is lagrangianLR), this process is shown to be computationally complex. It needs quantity that is big of to converge.

- Computationally Effective Bandwidth Allocation and energy Control for OFDMA:

Because the algorithm proposed by Wong et al. [5] wasComputationallyomplex that is $\mathrm{c}$ a computationally efficient bandwidth and energy control for OFDMA is proposedby Kivanc et al. [6] for downlink interaction. Their algorithm minimizes energy that is total while keeping information prices. They considered a cell that is straightforward one base station and several mobile channels. The assumption is that the Channel State Information (CSI) because of fading that is multipath well as pathloss and shadowing results are understood precisely at both transmitter and receiver, i.e., the channel $g$ ain for each subcarrier is thought to $b$ age understood. It's also thought that people have actually details about existence of a multiple access professional tocol which provides details about channel state, subcarrier allocation etc., to and through the base place and place that is mobile [40].

Right here, the subcarrier that is optimal energy allocation is split into two actions:

1. Resource Allocation: determine the amount of subcarriers each individual gets, i.e., its bandwidth, centered on price demands plus the users' normal channel gain.

2. Subcarrier Allocation: utilize the total results of the resourceallocation phase and hannel that is $\mathrm{c}$ nformation to allocate the subcarriers towards the users.

\section{Site Allocation Algorithm}

Some users will see a lesser general SNR than many other users in an invisible environment. These users have a tendency to need

The energy that is many. Learning the subcarrier allocations through the LR algorithm implies that as soon as users have sufficient subcarriers to meet their minimum price demands, giving more subcarriers to users with reduced normal SNR really helps to lessen the transmission energy that is total. So that they have actually

Proposed the bandwidth had been called by an algorithm projectconsidering SNR (BABS) algorithm which uses the SNR that is typical each individual to decide the amount of subcarriers that individual will likely to be assigned.

This algorithm discovers the circulation of subcarriers that minimizes the sum total energy needed whenever every individual experiences a channel that is flat-fading. 
Subcarrier Assignment Algorithms When the real wide range of subcarriers is determined, the alternative would be to designate subcarriers which can be certain users. Within the 2nd phase associated with the algorithm, the algorithm discovers $t$ he assignment that is better of subcarriers to users. Two various approaches presented within their paper are: the rate-craving algorithm that is greedy plus the amplitude-craving greedy algorithm ACG). The RCG algorithm starts with an estimate associated with the users' transmission price for each provider and aims to maximise the transmission price that is total. The ACG algorithm is an adjustment o f RCG which achieves comparable performance at reduced complexity that is computational.

Their algorithm is reported to own benefits such as for example less complexity, easy, achieves good performance also it enables efficient usage of system resources in terms of send energy, bandwidth effectiveness and time that is computational. Nevertheless the performance of the algorithm is based on just how quickly the channel differs.

- Subcarrier Allocation and B it Loading Algorithms for OFDMA-Based systems which can be cordless:

The writers of [6] provide computationally efficient algorithms for subcarrier and bit allocation in a cellular situation that is solitary. Now the problem is known as by us where extra constraints due to link that is interfering. In [7], the situation of assigning subcarriers and bits to point-to-point links being wireless the presence o $\mathrm{f}$ co-channel interference and Rayleigh diminishing is addressed. This process was proposed by Gautham Kulkarni et al. Right here, the authors concentrate on fixed infrastructure-less sites being cordless employ OFDMA once the MAC scheme. They've thought an idealized time and regularity system that is synchronous. The essential problems to be considered are location fading that is reliant and energy control. The goal of their work would be to reduce the sum total energy that is sent the whole community while satisfying the information price requirement of every website link.

The writers presented centralized algorithms for subcarrier and $b$ it allocation within the presence of interfering links. Centralized algorithms are employed once we know about the channel gains for whole system. The goal is to designate a couple of subcarriers every single load and website link bits on each assigned subcarrier. The facets to be considered would be the course that is frequency-dependent parameters together with disturbance as a result of other links which have been already assigned those subcarriers. Instead, this is viewed as recognition of sets of links that share each subcarrier. Whenever a hyperlink that is brand new assigned a specific subcarrier with a certain bit loading, the SINR thresholds for itself also a s for several other links currently making use of that subcarrier must $b$ age maintained. Energy control must be done to keep up the thresholds which are needed. The issue amounts to locating a suitable mapping for the bits o f each url to various subcarriers so that all SINR thresholds are maintained together with total energy that is sent a fter energy control, is minimal. W age present an algorithm that lots one little bit of a web link o nto a subcarrier at each and every action $t$ that is such the incremental transmitted energy after doing energy control is minimal. This technique is proceeded until either all bits from each website link happen s uccessfully packed onto some subcarrier or it is really not feasible to load any longer bits.

The SNRs during the receiver for every single subcarrier whereas the central algorithms need international knowledge of all channel gains since centralized algorithms need familiarity with the whole system topology and channel faculties of each website link, they've additionally proposed a distributed algorithm which calls for o nly regional knowledge, in other words. There would be no coordination along with other links. The node that $r$ uns the algorithm doesn't have familiarity with channel gains for the whole network - all it understands could be the state associated with the networks during the receiver in cases like this. The goal of these two algorithms is to meet the price requirements of each and every website link and also to reduce power. The distributed algorithm is sensible and easy. Frequency synchronization is $\mathrm{f}$ that is important the subcarriers in an OFDM system to stay orthogonal. Another problem is of timing control. I $n$ purchase for the OFDM that is FFT-based receiver function precisely, all signals must show up inside the guard period. The writers assumed an time that is idealized regularity synchronous system within their paper.

- Minimal Complexity Adaptive Energy and Subcarrier Allocation for OFDMA

We realize that the water concept that is pouringn'toffer fairness between your users when it comes to bitrates. Therefore a complexity that is low and subcarrier allocation algorithm for OFDMA was proposed by Ermolova et al. [8] that delivers fairness between your users with regards to bitrates as well as equivalent time it offer a substantial minimization associated with typical bit mistake price (BER) performance under fixed send energy. This process provides an adaptive (powerful) bit allocation where each subcarrier is packed according with $\mathrm{t}$ he CSI so as to optimize the functional system criterion such as for example minimization of $B$ ER or price maximization. The subcarriers experiencing deep diminishing cause detection mistakes in non-adaptive (static) techniques. The subcarrier that is adaptive method improves $t$ he system performance. The writers prove a lemma in regards to the inversion of energy gains within their paper and considering they recommend to augment the scheme with a complexity that is low loading algorithm that equalizes the $\mathrm{s}$ ignal to $\mathrm{n}$ oise ratio (SNR) for every individual [41].

Two algorithms of energy loading for OFDMA systems are proposed in this technique. The method that is first an expansion of the subcarrier allocation technique predicated on purchased data to your OFDMA. In bought subcarrier selection algorithm (OSSA), all subcarriers are bought in line with the values of these energy gains and just thestrongest subcarriers are similarly packed as the sleep ofthem are away from usage. How many the utilized subcarriers varies according to the modulation format adopted.

\section{RESULTS AND DISCUSSION}

Application of bought subcarrier allocation algorithm to OFDMA

In the scheme with the fixed subcarrier that is 
initial Allocation, the OSSA is used by each individual to your pair of L subcarriers allotted to this individual ahead of time individually associated with CSI. Each individual instructions the subcarriers based on the power gain values in the scheme utilizing the adaptive initial subcarrier allocation. Then users use the OSSA in a order that is random in other words. a randomly selected individual first employs the OSSA and then another randomly plumped for individual employs the OSSA and therefore do other people. If among the strongest subcarrier for the individual happens to be currently allotted to a different one, the next vacated bought subcarrier is assigned to the consumer. The random choice of initial individual additionally the equal quantity o $f$ the allocated subcarriers guarantees fairness when it comes to the throughput that is normal.

\section{Combination with energy loading}

The OSSA assumes energy that is equal for each subcarrier. It is obviously to expect that energy that would work loading $\mathrm{c}$ an improve that is further average BERperformance. The tested low complexity energy loading algorithm is founded on the lemma that is after.

Lemma: in every regularity selective radio channel, beginning with some transfer SNR value, the task of energy gain inversion constantly improves the BER that is average of the OFDMA system

- Effective Subcarrier, energy, and Rate Allocation with Fairness Consideration for OFDMA Uplink Another algorithm with fairness consideration is presented by Gao L. and Cui S. inside their paper [9]. Their algorithm is less complex also it maximizes the amount price under specific send and price energy constraints. Right Herefairness means confirmed target is immediately considered for several the users during subcarrier allocation to reduce outage. This algorithm achieves outage that is superior and its complexity depends linearly in the wide range of users and subcarriers. The algorithm first assigns the subcarriers to your users including two actions:

1. Subcarrier allocation: It further containsInitial subcarrier allocation, Recurring subcarrier allocation

In initial subcarrier allocation, subcarriers are assigned just towards the users whoever prices are underneath the target that is predefined. After initial subcarrier allocation is completed, then recurring subcarrier allocation is performed to help increase amount price if you may still find subcarriers unallocated.

1. Single individual energy and price allocation

After subcarrier allocation is completed, solitary individual optimal price and energy allocation is conducted for every single individual with water filling over assigned subcarriers. This algorithm is less complex and simple to implement and provides fairness that is high the users. But fairness that is increased ay decrease system throughput.

- Reduced Complexity Subcarrier Allocation Schemes for DFT-Precoded OFDMA Uplink Techniques

The downside that is primary of systems is its high. Peak-to-Average Energy Ratio (PAPR). OFDMA with Discrete Fourier Transform (DFT) precoding provides low PAPR regarding the sign that is sent. The subcarrier ALLOCATION ISSUE O F MAXIMIZING TOTAL RATES WHICH

CAN BE attainable multi- individual DFTprecoded OFDM uplink systems is examined by $\mathrm{P}$ ao W. and Chen Y. [10]. The absolute most updated algorithm till then had been proposed by Haustein et al. [11]. Their algorithm assigned energy that is equal subcarriers so that you can optimize total attainable prices. The algorithm had been hard to resolve due to power that is different of every individual. Additionally the algorithm has a higher complexity that is computational. Therefore a minimal complexity algorithm ended up being introduced to maximise total attainable prices that offer more performance compared to the one that's current. This algorithm makes use of the proposed user's efficiency that is spectral index to designate subcarriers to users. Initially, a subcarrier that is advised each individual is recognized as, after which the spectral effectiveness improvement indexes among users are thought. A person because of the greatest spectral effectiveness improvement index obtains the subcarrier that is recommended. Subcarriers are assigned to users one at a time in this way. Each step of the process regarding the proposed scheme is d esigned to maximise the full total price that is attainable. They will have additionally offered an subcarrier that is iterative scheme which further improves performance at the cost of increased complexity.

\section{REFERENCES}

1. KaliyamurthieKumarave A., RangarajanK.,Algorithm for automaton specification for exploring dynamic labyrinths,Indian Journal of Science and Technology,V-6,I-SUPPL5,PP-4554-4559,Y-2013

2. P. Kavitha, S. Prabakaran "A Novel Hybrid Segmentation Method with Particle Swarm Optimization and Fuzzy C-Mean Based On Partitioning the Image for Detecting Lung Cancer" International Journal of Engineering and Advanced Technology (IJEAT) ISSN: 2249-8958, Volume-8 Issue-5, June 2019

3. Kumaravel A., MeeteiO.N.,An application of non-uniform cellular automata for efficient cryptography,2013 IEEE Conference on Information and Communication Technologies, ICT 2013,V-,I-,PP-1200-1205,Y-2013

4. Kumarave A., RangarajanK.,Routingalogrithm over semi-regular tessellations,2013 IEEE Conference on Information and Communication Technologies, ICT 2013,V-,I-,PP-1180-1184,Y-2013

5. P. Kavitha, S. Prabakaran "Designing a Feature Vector for Statistical Texture Analysis of Brain Tumor" International Journal of Engineering and Advanced Technology (IJEAT) ISSN: 2249-8958, Volume-8 Issue-5, June 2019

6. Dutta P., Kumaravel A.,A novel approach to trust based identification of leaders in social networks,Indian Journal of Science and Technology,V-9,I-10,PP--,Y-2016

7. Kumaravel A., DuttaP.,Application of Pca for context selection for collaborative filtering,Middle - East Journal of Scientific Research,V-20,I-1,PP-88-93,Y-2014

8. Kumaravel A., RangarajanK.,Constructing an automaton for exploring dynamic labyrinths,2012 International Conference on Radar, Communication and Computing, ICRCC 2012,V-,I-,PP-161-165,Y-2012

9. P. Kavitha, S. Prabakaran "Adaptive Bilateral Filter for Multi-Resolution in Brain Tumor Recognition" International Journal of Innovative Technology and Exploring Engineering (IJTTEE) ISSN: 2278-3075, Volume-8 Issue-8 June, 2019

10. KumaravelA.,Comparison of two multi-classification approaches for detecting network attacks, World Applied Sciences Journal,V-27,I-11,PP-1461-1465,Y-2013

11. Tariq J., KumaravelA.,Construction of cellular automata over hexagonal and triangular tessellations for path planning of multi-robots,2016 IEEE International Conference on Computational Intelligence and Computing Research, ICCIC 2016,V-,I-,PP--,Y-2017

12. Sudha M., KumaravelA.,Analysis and measurement of wave guides using poissonmethod,Indonesian Journal of Electrical Engineering and Computer Science,V-8,I-2,PP-546-548,Y-2017

13. Ayyappan G., Nalini C., KumaravelA.,Various approaches of knowledge transfer in academic social network,International Journal of Engineering

and

ished By: 


\section{Subcarrier Allocation Techniques In Ofdma}

Technology,V-,I-,PP-2791-2794,Y-2017

14. Kaliyamurthie, K.P., Sivaraman, K., Ramesh, S. Imposing patient data privacy in wireless medical sensor networks through homomorphic cryptosystems 2016, Journal of Chemical and Pharmaceutical Sciences 92.

15. Kaliyamurthie, K.P., Balasubramanian, P.C. An approach to multi secure to historical malformed documents using integer ripple transfiguration 2016 Journal of Chemical and Pharmaceutical Sciences 92

16. A.Sangeetha,C.Nalini,"Semantic Ranking based on keywords extractions in the web", International Journal of Engineering \& Technology, 7 (2.6) (2018) 290-292

17. S.V.GayathiriDevi,C.Nalini,N.Kumar,"An efficient software verification using multi-layered software verification tool "International Journal of Engineering \& Technology, 7(2.21)2018 454-457

18. C.Nalini,ShwtambariKharabe,"A Comparative Study On Differen Techniques Used For Finger - Vein Authentication", International Journal Of Pure And Applied Mathematics, Volume 116 No. 82017 , 327-333, Issn: 1314-3395

19. M.S. Vivekanandan and Dr. C. Rajabhushanam, "Enabling Privacy Protection and Content Assurance in Geo-Social Networks", International Journal of Innovative Research in Management, Engineering and Technology, Vol 3, Issue 4, pp. 49-55, April 2018.

20. Dr. C. Rajabhushanam, V. Karthik, and G. Vivek, "Elasticity in Cloud Computing", International Journal of Innovative Research in Management, Engineering and Technology, Vol 3, Issue 4, pp. 104-111, April 2018

21. K. Rangaswamy and Dr. C. Rajabhushanamc, "CCN-Based Congestion Control Mechanism In Dynamic Networks", International Journal of Innovative Research in Management, Engineering and Technology, Vol 3, Issue 4, pp. 117-119, April 2018.

22. Kavitha, R., Nedunchelian, R., "Domain-specific Search engine optimization using healthcare ontology and a neural network backpropagation approach", 2017, Research Journal of Biotechnology, Special Issue 2:157-166

23. Kavitha, G., Kavitha, R., "An analysis to improve throughput of high-power hubs in mobile ad hoc network", 2016, Journal of Chemical and Pharmaceutical Sciences, Vol-9, Issue-2: 361-363

24. Kavitha, G., Kavitha, R., "Dipping interference to supplement throughput in MANET" , 2016, Journal of Chemical and Pharmaceutical Sciences, Vol-9, Issue-2: 357-360

25. Michael, G., Chandrasekar, A.,"Leader election based malicious detection and response system in MANET using mechanism design approach", Journal of Chemical and Pharmaceutical Sciences(JCPS) Volume 9 Issue 2, April - June 2016

26. Michael, G., Chandrasekar, A.,"Modeling of detection of camouflaging worm using epidemic dynamic model and power spectral density", Journal of Chemical and Pharmaceutical Sciences(JCPS) Volume 9 Issue 2, April - June 2016

27. Pothumani, S., Sriram, M., Sridhar, J., Arul Selvan, G., Secure mobile agents communication on intranet,Journal of Chemical nd Pharmaceutical Sciences, volume 9, Issue 3, Pg No S32-S35, 2016

28. Pothumani, S., Sriram, M., Sridhar, Various schemes for database encryption-a survey, Journal of Chemical and Pharmaceutical Sciences, volume 9, Issue 3, Pg NoS103-S106, 2016

29. Pothumani, S., Sriram, M., Sridhar, A novel economic framework for cloud and grid computing, Journal of Chemical and Pharmaceutical Sciences, volume 9, Issue 3, Pg No S29-S31, 2016

30. Priya, N., Sridhar, J., Sriram, M. "Ecommerce Transaction Security Challenges and Prevention Methods- New Approach" 2016 ,Journal of Chemical and Pharmaceutical Sciences, JCPS Volume 9 Issue 3.page no:S66-S68

31. Priya, N.,Sridhar,J.,Sriram, M."Vehicular cloud computing security issues and solutions" Journal of Chemical and Pharmaceutical Sciences(JCPS) Volume 9 Issue 2, April - June 2016.

32. Priya, N., Sridhar, J., Sriram, M. "Mobile large data storage security in cloud computing environment-a new approach" JCPS Volume 9 Issue 2. April - June 2016

33. Anuradha.C, Khanna.V, "Improving network performance and security in WSN using decentralized hypothesis testing "Journal of Chemical and Pharmaceutical Sciences(JCPS) Volume 9 Issue 2, April - June 2016

34. Anuradha.C, Khanna.V, "A novel gsm based control for e-devices" Journal of Chemical and Pharmaceutical Sciences(JCPS) Volume 9 Issue 2, April - June 2016

35. Anuradha.C, Khanna.V, "Secured privacy preserving sharing and data integration in mobile web environments " Journal of Chemical and Pharmaceutical Sciences(JCPS) Volume 9 Issue 2, April - June 2016.
36. Sundarraj, B., Kaliyamurthie, K.P. Social network analysis for decisive the ultimate classification from the ensemble to boost accuracy rates 2016 International Journal of Pharmacy and Technology 8

37. Sundarraj, B., Kaliyamurthie, K.P. A content-based spam filtering approach victimisation artificial neural networks 2016 International Journal of Pharmacy and Technology 83.

38. Sundarraj, B., Kaliyamurthie, K.P. Remote sensing imaging for satellite image segmentation 2016 International Journal of Pharmacy and Technology 83.

39. Sivaraman, K., Senthil, M. Intuitive driver proxy control using artificial intelligence 2016 International Journal of Pharmacy and Technology 84.

40. Sivaraman, K., Kaliyamurthie, K.P. Cloud computing in mobile technology 2016 Journal of Chemical and Pharmaceutical Sciences 9 2.

41. Sivaraman, K., Khanna, V.Implementation of an extension for browser to detect vulnerable elements on web pages and avoid click jacking 2016 Journal of Chemical and Pharmaceutical Sciences 92.

\section{AUTHORS PROFILE}

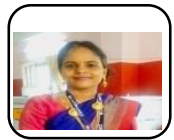

G.Kavitha Assistant Professor, Department of Computer Science \&Engineering, Bharath Institute of Higher Education and Research, Chennai, India

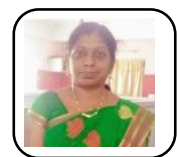

N.Priya, Assistant Professor, Department of Computer Science \& Engineering, Bharath Institute of Higher Education and Research, Chennai, India

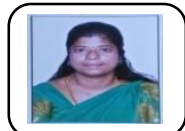

R.Velvizhi, Assistant Professor, Department of Computer Science \& Engineering, Bharath Institute of Higher Education and Research, Chennai, India 